\title{
Arbeitszeitbedarf von aktuellen Gruppenhaltungsverfahren des Wartebereichs in konventioneller Zuchtsauenhaltung in Österreich
}

\section{Working time requirement of current group keeping systems in the waiting area of conventional breeding sow husbandry in Austria}

\author{
Elisabeth Quendler ${ }^{1}$, Robert Kaufmann ${ }^{1}$, Matthias Schick ${ }^{2}$
}

\author{
${ }^{1}$ Universität für Bodenkultur, Department für Nachhaltige Agrarsysteme, Institut für Landtechnik, Peter-Jordan-Straße 82, 1190 Wien, \\ Österreich \\ ${ }^{2}$ Strickhof, Tierhaltung \& Milchwirtschaft, Eschikon 21, 8315 Lindau, Schweiz \\ Korrespondierende Autorin: elisabeth.quendler@boku.ac.at
}

Einreichung: 13. August 2020, überarbeitete Einreichung: 26. März 2021, Annahme: 29. März 2021

\begin{abstract}
Zusammenfassung
In der konventionellen Zuchtsauenhaltung ist es wichtig, die verfügbare Arbeitszeit so effizient wie möglich zu nutzen, um den größtmöglichen einzelbetrieblichen Erfolg unter Einhaltung rechtlicher Produktionsvorgaben sicherzustellen. Dies kann durch das Bereitstellen von aktuellen Planungsdaten in Form von Arbeitszeitbedarfswerten unterstützt werden. Ziel dieser Studie war daher, Arbeitszeitdaten von aktuellen Gruppenhaltungsverfahren des Wartebereichs in der konventionellen Zuchtsauenhaltung zu generieren. Dafür wurden bei den Haltungsverfahren Kleingruppenhaltung mit Fressliegebucht und Großgruppenhaltung mit Abrufstation eine Datenerhebung mit mobiler Videotechnik und halbstandardisierten Fragebogen an vier landwirtschaftlichen Betrieben mit aktueller Technik durchgeführt. Mit den daraus gewonnenen Planzeiten wurden unter optimierten Bedingungen Betriebsmodelle erstellt, um für verschiedene Betriebsgrößen, den Arbeitszeitbedarf von Haltungsverfahren und Arbeitsvorgängen zu modellieren und darzustellen. Beide Haltungsverfahren unterschieden sich teilweise im Arbeitszeitbedarf nach Arbeitsvorgängen sowie im Gesamtarbeitszeitbedarf bei verschiedenen modellierten Bestandsgrößen signifikant voneinander. Der Gesamtarbeitszeitbedarf im Wartebereich bei einer Bestandsgröße von 140 Zuchtsauen lag bei der Kleingruppe bei 3,58 AKh/Sau/Jahr und bei der Großgruppe bei 2,68 AKh/Sau/Jahr. Gegenüber Ergebnissen älterer Studien belegte sich der Trend einer Abnahme des Arbeitszeitbedarfs je Sau und Jahr. Diesen bedingte vorwiegend der technische Fortschritt, belegt über die Arbeitszeitbedarfsunterschiede nach eingesetzter Technik, und hiermit ermöglichten größeren Bestandsgrößen.
\end{abstract}

Schlagwörter: Zuchtsauenhaltung, Arbeitszeitbedarf, Wartestall, Fressliegebucht, Abrufstation

\section{Summary}

In keeping of breeding sows, it is important to use the working time as efficiently as possible to ensure economics within legal production conditions. This can be supported by providing working time requirement data. Therefore, the aim of this study was to generate working time requirement of current group keeping systems in the waiting area of breeding sows. For this purpose, in the keeping systems for small groups with feeding bay and large groups with retrieval station, data was collected using mobile video technology and semi-standardized questionnaires at four farms with current technology. With the gained data, operating models were created under optimized conditions in order to display the working time requirement of work processes for different stock sizes. Both keeping systems differed significantly from each other in the total working time requirement over different stock sizes. The total working time requirement in the waiting area with a typical herd size of 140 breeding sows of Austrian growing farms was $3.58 \mathrm{MPh} /$ sow/year for the small group and $2.68 \mathrm{MPh} /$ sow/year for the large group. The comparison of the available results with data from previous studies indicated the trend of decrease in working hours per sow and year. This was due to technical progress which enabled larger herd sizes.

Keywords: breeding sow, keeping system, working time requirement, waiting room, feeding bay, feeding station 


\section{Abkürzungen/Abbreviations}

AKcmin: Arbeitskraft-Zentiminuten (=1/100 AKmin); MPcmin: Man power centiminutes

AKh: Arbeitskraftstunde; MPh: Man power hours

APmin: Arbeitspersonenminuten; MPmin: Man power minutes

BM: Bezugsmenge; Reference quantity

$\mathrm{cmin}$ : Centiminute $(1 \mathrm{cmin}=1 / 100 \mathrm{~min})$ : Centiminute

t: Zeit

3-FL-SFB: 3-Flächen-Selbstfangstand

GH-SG: Gruppenhaltung - stabile Gruppe

\section{Problemstellung}

Die Ferkelproduktion in Österreich ist gefordert, bei steigenden Energie- und Rohstoffpreisen sowie stagnierenden Erzeugerpreisen und höheren Tierwohlauflagen im globalen Wettbewerb zu bestehen. So kam es, dass in den vergangenen zwei Jahrzehnten viele Betriebe entweder aus der Produktion ausstiegen oder Betriebsvergrößerungen vornahmen. Durch Investitionen wurden zunehmend mehr Gruppenhaltungs- und automatisierte sowie effizientere Arbeitsverfahren auf den ferkelproduzierenden Betrieben implementiert (Statistik Austria, 2018, Hoy et al., 2015, Martel et al., 2008). Das EU-weite Gebot der Gruppenhaltung tragender Sauen seit 2013 bedingte gravierendste Veränderungen in der Schweinehaltung sowie forcierte den Strukturwandel (Hoy et al., 2015). Moderne Arbeitsverfahren und Haltungssysteme sowie strenge rechtliche Vorgaben beeinflussen folglich erheblich den Arbeitszeitaufwand und die Arbeitserledigungskosten. Arbeitszeitbedarfswerte spielen für einzelbetriebliche Planungen eine maßgebliche Rolle. In der Literatur sind gegenwärtig keine aktuellen Arbeitszeitbedarfswerte für den Wartebereich zu finden. Die vorhandenen aktuellsten mittleren Arbeitszeitbedarfswerte belaufen sich für den Wartebereich der Großgruppenhaltung auf Tiefstreu ab 1,60 AKh (ohne Managementaufwand) bis 34,4 AKh/Sau/Jahr über alle Haltebereiche für österreichische Verhältnisse (Handler et al., 2006; Quendler et al., 2010). Untereinander sind die derzeit vorliegenden Studien nur schwer zu vergleichen, da unterschiedliche Bestandsgrößen, Haltebereiche, Haltungssysteme und Wirtschaftsweisen zu Grunde liegen. Nicht immer besteht eine klare Darlegung des Arbeitszeitbedarfs nach den einzelnen Stallbereichen (Abferkel-, Warte- und Ferkelaufzuchtbereich) sowie Haltungssystemen (Handler et al., 2006, Haidn et al., 2007, Riegel et al., 2006, Quendler et al., 2010, Quendler et al., 2013).

Für das Halten von Zuchtsauen gibt es derzeit mehrere Möglichkeiten, die von sechs wesentlichen Faktoren, stabile oder dynamische Gruppen, Gruppengröße, Anzahl der Funktionsbereiche und Art des Fütterungssystems sowie Vorhandensein oder Nichtvorhandensein von Auslauf sowie Einstreu, bestimmt sind. Diese Faktoren sind mehr oder weniger voneinander abhängig und beeinflussen das jeweilige Haltungssystem sowie bestimmen wesentlich die Arbeitsverfahren und den Arbeitszeitaufwand oder -bedarf mit. Je nach Verfügbarkeit von Arbeitskraft, Einstreu, Betriebstyp und -ziel werden die Arbeitsverfahren, abgestimmt auf das Haltungssystem, vom Betriebsleiter gewählt. In der konventionellen österreichischen Ferkelproduktion werden die Sauen im Wartebereich überwiegend in 2-Flächenbuchten in Kleingruppen oder in 3-Flächenbuchten ohne Auslauf und Einstreu in Großgruppen gehalten. Haltungssysteme mit Auslauf und Einstreu sind in Label- und Biobetrieben vorzufinden. Die Differenzierung in Groß- und Kleingruppe hängt nicht nur von der Anzahl der Sauen, sondern auch deren Zusammensetzung nach Reproduktionsstadien ab. Eine Großgruppe zeichnet sich durch Sauengruppen mit deutlich mehr als 20 Sauen aus, deren Zusammensetzung öfter durch das Ein- und Ausstallen von Sauen, die in verschiedenen Reproduktionsstadien stehen, wechseln. Die deutsche Tierschutznutztierhaltungsverordnung differenziert in drei Gruppen, eine Kleingruppe mit bis zu 5 Stück, die mittlere Gruppe bis 39 Stück und die Großgruppe ab 40 Stück Zuchtsauen (Weiß et al., 2005, Ziron, 2010, Ziron et al., 2018). Die Kleingruppe ist durch eine gute Bestandsübersicht geprägt, Nachteile ergeben sich bei der Strukturierung, über die mangelnde Ausweichmöglichkeit und Notwendigkeit der Sortierung nach Kondition. Deren Buchten sind als 2- oder 3-Flächenbucht ausgestaltet. Die 2-Flächenbucht ist in zwei strukturierte Bereiche aufgeteilt. Zum einen in einen Fressund Liegebereich und zum anderen in einen Aktivitätsbereich. Der Fressbereich ist durch Fressstände untergliedert und dient auch als Liegebereich. Im Aktivitätsbereich ist Beschäftigungsmaterial vorhanden und dieser dient gleichzeitig als Kotbereich. Eine 3-Flächenbucht ist in einen Fress-, einen Liege- und einen Aktivitätsbereich gegliedert (Ziron, 2010). Bei der Großgruppenhaltung werden die Sauen in einer Abrufstation einzeln mit Trockenfutter oder gruppenweise mit Flüssigfütterung gefüttert. Der Liegebereich ist in mehrere Buchten mit drei umseitig geschlossenen Wänden unterteilt. Der Aktivitätsbereich ist auch der 
Kotbereich (Weiß et al., 2005; ÖKL, 2016, Ziron, 2010). Beim Fütterungssystem ist zwischen gleichzeitigem Fressen der Tiere am Trog (Fressstand, Fressplatzteiler) und nicht gleichzeitigem Fressen, einer Abruffütterung, einer rationierten individuellen Fütterung, zu unterscheiden (Hoy et al., 2015, Ziron et al., 2018). Je nach Haltungssystem und Betriebstyp kann ein Auslauf angegliedert sein und Einstreu verwendet werden sowie variieren die Arbeitsverfahren und folglich der Arbeitszeitaufwand sowie -bedarf (Weiß et al., 2005; Riegel et al., 2009). Das Auftreten von Leistungsminderungen durch höhere Umrauscherrate, niedrigere Wurfgröße sowie von gesundheitlichen Schäden, bedingt durch Rangkämpfe, kann bei Gruppenhaltung durch gutes Management erheblich verhindert werden. Der günstigste Zeitpunkt der Gruppenbildung ist unmittelbar nach dem Absetzen der Ferkel von den Sauen gegeben. Die Tiere sind nicht tragend und die Rangordnungskämpfe können keinen Schaden an der Trächtigkeit anrichten (Hoy et al., 2015).

Zum Erfassen von Arbeitszeiten kamen in bisherigen sehr ähnlichen Studien finale und kausale Methoden zum Einsatz (Haidn et al., 2007; Riegel et al., 2009). Beim Einsatz dieser Methoden wurden die Arbeitszeiten durch Erfragen des Arbeitszeitaufwandes oder messtechnisches Erfassen des Arbeitszeitbedarfs von Arbeitsteilabschnitten ermittelt. Die Gesamtarbeit wurde in Arbeitsvorgänge bis -elemente für das Erheben aufgegliedert. Die finalen Methoden, die eingesetzt wurden, generieren erfragte und dokumentierte Schätzwerte in Arbeitstagebüchern von Beschäftigten auf den Betrieben (Riegel und Schick, 2006). Das Gliederungsniveau der kausalen Erhebungsmethoden entspricht der kleinsten Gliederungsebene der Gesamtarbeit der Arbeitszeitanalyse sowie Arbeitszeitelementmethode nach Auernhammer (1976). Diese Methoden basieren auf direkten (Arbeitzeitnehmer mit Zeitmesssoftware) oder indirekten Messungen (Videoaufzeichnung) in Untersuchungsbetrieben oder auf Arbeitsversuchen und eignen sich für Ist-Analysen, Planzeiterstellungen und Soll-IstVergleiche. Die bisher durchgeführten Arbeitszeitstudien der Zuchtsauenhaltung, die für diese Studie von Bedeutung sind, unterschieden sich einerseits in der Gliederungsintensität der Gesamtarbeit für das Ausweisen des Arbeitszeitaufwands sowie -bedarfs und in der eingesetzten analogen und digitalen Erhebungstechnik. Andererseits bestanden Unterschiede in der Kategorisierung der Tätigkeiten in tägliche und nicht tägliche sowie Routine- und Sonderarbeiten als auch Kontrolltätigkeiten und Managementaufgaben (Haidn, 1992; Handler et al., 2006; Haidn et al., 2007; Riegel et al., 2009; Quendler et al., 2010; Quendler et al., 2013). Die beiden letztgenannten Tätigkeitsbereiche etablierten sich mit der Automatisierung und dem Entstehen von größeren Betriebseinheiten. In diesem Zusammenhang legten Riegel und Schick (2006) drei Hauptkriterien, die auf den Gesamtarbeitszeitbedarf wirken, fest. Dies sind die Bestandsgröße, das Haltungsverfahren und der Mechanisierungsgrad des Betriebs. Aufgrund der verschiedenen Gliederungsniveaus der Gesamtarbeit, Kategorisierung der Tätigkeiten und Stallbereiche sind die ermittelten Ergebnisse zu Arbeitszeitaufwand und -bedarf teils schwierig vergleichbar.

Folglich wurde als Ziel in dieser Studie das Ermitteln von Arbeitszeitdaten von aktuellen Gruppenhaltungsverfahren des Wartebereichs in der konventionellen Zuchtsauenhaltung in Österreich, Kleingruppe mit Fressliegebuchten und Großgruppe mit Abrufstation, verfolgt. Die aktuellen Arbeitsverfahren waren zu erheben, zu beschrieben, deren Einflussgrößen und Arbeitszeitbedarfswerte zu ermitteln, Unterschiede zwischen Haltungsverfahren für Modellbetriebe darzulegen und arbeitswirtschaftliche Kennzahlen für die Praxis zu erarbeiten.

\section{Material und Methoden}

Als Untersuchungsbetriebe wurden Zuchtsauen haltende Betriebe im österreichischen Hauptproduktionsgebiet Südöstliches Flach- und Hügelland, welche eine Datenerhebung mit Videotechnik am Betrieb ermöglichten, Klein- oder Großgruppenhaltung mit stabilen Gruppen gemäß dem aktuellen Stand der Technik im Wartestall hatten und nach guter fachlicher Praxis produzierten, ausgewählt. Die Datenerhebung fand auf den vier ausgewählten Untersuchungsbetrieben zwischen 2017 und 2018 statt (Tabelle 1). Die kleine Stichprobengröße ermöglichte die detaillierte Aufgliederung der Arbeit auf das Niveau von Arbeitselementen. Für ein zuverlässiges Ermitteln von Planzeiten von bisher unbekannten Arbeitselementen sind $2^{\mathrm{n}}$ Messungen auf der kleinsten Gliederungsebene nötig (Auernhammer, 1976). Die Zuverlässigkeit der modellierten Ergebnisse auf Arbeitsvorgangsebene wurde über den Daten- und Modellierungsabgleich mit der Arbeitselementdatenbank PROOF sichergestellt (Schick, 2005). Die Untersuchungsbetriebe hielten zwischen 70 und 200 Zuchtsauen (Mittelwert (MW): 120, Standardabweichung (SD): 63) pro Betrieb. Es wurden zwischen 270 bis 850 Stück Ferkel (MW: 548, SD: 305) in der 
Tabelle 1. Haltungs-, Fütterungs- und Entmistungssysteme im Wartebereich der Untersuchungsbetriebe $(\mathrm{n}=4)$

Table 1 . Housing, feeding and manure systems in waiting area of farms examined $(n=4)$

\begin{tabular}{lccccc}
\hline Systeme & Bereich & Betrieb 1 & Betrieb 2 & Betrieb 3 & Betrieb 4 \\
\hline Haltungssystem & DZ & 3-FL-SFB & BKB & BKB & GH-SG \\
Fütterungsystem & W & 3-FL-SFB & 3-FL-SFB & RBTF & Spotmix - \\
& WS & RBTF & RBTF & Spotmix - \\
ARS & TS / Gülle & TS / Gülle \\
Entmistungssystem & RBTF & TS / Gülle & TS / Gülle \\
\hline
\end{tabular}

Ferkelaufzucht jährlich produziert. Im Mittel wurden 2,45 Würfe (SD: 0,08) je Sau und Jahr sowie 27,0 Ferkel je Wurf, bei durchschnittlich 26,3 Säugetagen, abgesetzt. Bei Betrieb 1 und 2 wurden die Zuchtsauen in Kleingruppen zu je 5 Tieren gehalten und der Zuchtsauenbestand belief sich auf jeweils 70 Stück.

Bei Betrieb 3 und 4 wurden die Zuchtsauen in stabilen Gruppen zu je 40 beziehungsweise 35 Tieren in Großgruppen geführt, wobei sich deren Bestand auf 200 beziehungsweise 140 Stück belief. Auf Betrieb 1 war das Haltungssystem im Deckzentrum (DZ) und im Wartestall (WS) und auf Betrieb 2 im WS eine 3-Flächen-Selbstfangbucht (3-FL-SFB). Das DZ der Betriebe 2 bis 4 hatte Besamungskorbbuchten (BKB). Als Fütterungssysteme wurden im Betrieb 1 und $2 \mathrm{im} \mathrm{DZ}$ und WS sowie am Betrieb 3 im DZ eine Rohrbahn-Trockenfütterung (RBTF) mit Volumendosierer verwendet. Im Betrieb 4 wurde das Futter im DZ mit einer Spotmix-Anlage zugeteilt. Auf Betrieb 3 und 4 wurden die Zuchtsauen im WS mit einer Abrufstation (ARS) gefüttert, welche durch eine Spotmix-Anlage befüllt wurde. Betrieb 1 hatte als Entmistungssystem einen Vollspaltenboden (VS) mit Güllesystem im DZ und WS. Betrieb 2, 3 und 4 nutzten als Entmistungssystem einen Teilspaltenboden (TS) mit Güllesystem im DZ und WS. Die Stallgebäude von allen Betrieben befanden sich in einen baulich guten Zustand und wurden zwischen 1998 und 2013 errichtet oder renoviert und modernisiert (MW: 13,1 Jahre, SD: 4,04).

Zur Feststellung der Arbeitsverfahren und des Arbeitszeitaufwands sowie -bedarfs im Wartebereich wurden einerseits die Befragung mit einem halbstandardisierten Fragebogen als finale Methode und andererseits die Arbeitsbeobachtung mit indirekter Messung als kausale Methode verwendet. Der halbstandardisierte Fragebogen wurde zur Ermittlung von Schätzwerten für Arbeitsvorgänge von Arbeiten eingesetzt, die jährlich nicht sehr oft vorkamen sowie messtechnisch schwierig erhebbar waren. Für das indirekte Messen wurde die mobile Videotechnik, auf Ebene des Arbeitselementes, angewendet. Diese wurde gewählt, um diese einerseits methodisch in der konventionellen Zuchtsauenhaltung $\mathrm{zu}$ erproben, da bis dato nur die analoge eingesetzt wurde (Quendler et al., 2007). Das Aufzeichnen wurde mit der Digitalkamera SONY DSC-HX50 und einer 32 Gigabit Speicherkarte vorgenommen. Auf allen Untersuchungsbetrieben wurden die Arbeitszeiten von Routine- und Sonderarbeiten und deren Einflussgrößen von einem Produktionszyklus in 2 bis 5 Aufnahmetagen je Betrieb erhoben. Die Arbeitsvorgänge und deren Arbeitselemente mit den Arbeitszeiten der IST-Situation, dokumentiert in den aufgenommenen Videos, wurden mit der Zeitmesssoftware ORTIMzeit 6.5 nach der Arbeitselementmethode nach Auernhammer (1976) ausgewertet. Im Tabellenkalkulationsprogramm (Excel) erfolgte die deskriptive und in SAS Enterprise Guide 7.1 und Regressa 5.0 die analytische Auswertung für die Generierung von statistisch abgesicherten Planzeiten (DMC, 2019). Prüfkriterien zur Absicherung der Datengüte der ermittelten arbeitselementbezogenen Mittelwerte waren der Variationskoeffizient bei nicht zyklischen und das Epsilon bei zyklischen Arbeitselementen (Fricke, 2004). Zum Nachweis von signifikanten Unterschieden im Arbeitszeitbedarf von systembezogenen Planzeitelementen wurde die einfaktorielle Varianzanalyse mit anschließender Bonferroni-Korrektur und Gewichtung nach Welch nach Quendler et al., (2010) eingesetzt. Die Werte unterschieden sich voneinander, wenn die Irrtumswahrscheinlichkeit kleiner als $5 \%(\mathrm{p}<0,05)$ war. Je Klein- und Großgruppe wurde ein Modellbetrieb mit einem Sauenbestand von 140 Zuchtsauen unter optimierten Bedingungen im 
Wartebereich modelliert. Um für verschiedene Bestandsgrößen, typische Bestandsgrößen der Praxis im deutschsprachigen Raum von 70 bis 1050 Zuchtsauen, Arbeitszeitbedarfswerte je Arbeitsvorgang und Haltungssystem berechnen zu können, wurden die Modelle in das Modellkalkulationssystem PROOF Schweine (Schick, 2005) integriert und Regressionsgleichungen in Regressa 5.0 erstellt und auf Plausibilität evaluiert.

\section{Ergebnisse und Diskussion}

In diesem Abschnitt sind einerseits die Ergebnisse zur erstmalig eingesetzten digitalen Datenerhebungstechnik, andererseits jene zum modellierten Arbeitszeitbedarf für das Halten von leeren und tragenden Zuchtsauen der untersuchten beiden Haltungssysteme beschrieben und diskutiert.

\subsection{Methodik}

Die mobile digitale Videotechnik zur Erhebung von Arbeitszeiten wurde erstmalig auf vier konventionellen Schweine haltenden Untersuchungsbetrieben eingesetzt. Bei der Auswahl von Betrieben für eine Datenerhebung stellte sich heraus, dass landwirtschaftliche Betriebe, aufgrund der Verwendung von Videotechnik im Versuch, wenig Bereitschaft für ein Mitwirken an dieser Studie zeigten. Nur jeder achte angefragte Betrieb sah sich für diese Studie als geeignet an. Der Großteil der Betriebsleiter hatte Skepsis bei einer Aufnahme von Videos in ihrem Betrieb. Genannte Gründe dafür waren das Alter der Stallgebäude, der Stand der technischen Einrichtung, Bedenken zur Hygiene als auch Aspekte des Tierschutzes im Sinne einer Veröffentlichung von Videos oder Bildern. Als Hindernis wurden auch zu komplexe und spezifische Forschungsergebnisse wahrgenommen. Fontana et.al. (2016) eruierte fehlende zeitliche sowie finanzielle Ressourcen der Zivilgesellschaft als Gründe für eine geringe Bereitschaft mit der Wissenschaft zusammen zu arbeiten.

Bei Auswertung von Vorversuchsvideos wurde festgestellt, dass ein Abstand von zwei bis drei Metern zur arbeitenden Person ideal ist, um sicherzustellen, dass die arbeitenden Hände immer gut sichtbar sind und um Anfang und Ende eines Arbeitselementes genau eingrenzen zu können und sich die arbeitende Person nicht gestört fühlt. Der Arbeitszeitaufwand für die Auswertung in der Zeitmessauswertesoftware ORTIMzeit 6.5 nahm nochmals die Dauer der Arbeitsbeobachtung in Anspruch. Der Einsatz dieser Methode gegenüber der Zeitnehmermessung mit der digitalen Zeitmesssoftware bedingte die doppelte Beobachtungszeit. Erste statistische Parameter zur Güte der Messwerte wurden erst im Zuge der Videoauswertung in ORTMzeit 6.5 ersichtlich. Es waren keine Mittelwerte oder ein erforderlicher Stichprobenumfang während der Arbeitsbeobachtung, wie bei der Zeitnehmermessung, gegeben. Nach Martetschläger (2007) und Pötz (2012) waren bei Verwendung eines Pocket-PCs oder eines Smartphones zur Arbeitszeitdatenerhebung die direkt gemessenen Zeitwerte während der Arbeitsbeobachtung und eine Kurzstatistik dazu einsehbar, die nach der Messung mit einer Synchronisierungssoftware direkt auf dem PC übertragen wurden. Folglich ist eine Datenerhebung mit einem Pocket-PC oder Smartphone zeitsparender durchführbar, jedoch kann nicht während der Messung die Arbeitsbeobachtung, wie im Video, gestoppt, verlangsamt abgespielt oder ein Arbeitselement oder ein Arbeitsteilvorgang nochmals gemessen oder für ergonomische Fragestellungen abgespielt werden. Mit der Erstellung von Videos sind relativ große Datenmengen zu bearbeiten und auf geeigneter Hardware zuverlässig zu speichern und gegen Verlust zu sichern.

In der Literatur gibt es einige Hinweise zu analoger und digitaler videobasierter Datenerhebung, insbesondere zu deren Vorteilhaftigkeit. Nach Haidn (1992) und Matetschläger (2007) sollte die analoge Videotechnik aufgrund der Erhebungsgenauigkeit und Kombinierbarkeit mit ethologischen Versuchen in der Tierhaltung bevorzugt gewählt werden. Wenngleich diese vor 20 Jahren im Vergleich zu Stoppuhr, Aufnahmeblatt oder Arbeitstagebuch in der Anschaffung sehr teuer war, wurde die videobasierte Arbeitszeiterfassung bis dato in der Landwirtschaft wenig genutzt. Quendler et al. (2007) wählten die festinstallierte digitale Videotechnik, um buchtspezifische Arbeiten verschiedener Abferkelbuchten sowie deren Arbeitszeitbedarf zu erheben. Mit dem videobasierten digitalen Erfassen der buchtbezogenen Arbeitszeit nach der Arbeitszeitelementmethode, mittels verkabelter digitaler Videoanlage, wurde eruiert, das nach mehrtägiger Anwendung dieser, kein Zeitnehmereinfluss mehr vorliegt und kontinuierliches sowie wiederholtes Messen und eine praktikable Auswertung in Kombination mit Auswertemakros im Tabellenkalkulationsprogramm gegeben ist. Sie führten dies an einen Gewöhnungseffekt der arbeitenden Person an Videokameras zurück. Beim Einsatz einer mobilen Kamera in dieser Studie kam es nach einer Gewöhnungsphase der arbeitenden Person zur gefilmten Arbeitssituation zu einer 
ähnlichen Situation. Es wurde kein Einfluss durch die filmende Person auf die arbeitende Person und deren Arbeitsgeschwindigkeit nach einer Gewöhnungsphase festgestellt. Sie erwies sich während der voranschreitenden Arbeitserledigung als praktikabel. Haidn (1992) eruierte zusätzlich, dass ein geringerer Arbeitszeitaufwand in Anwesenheit eines Zeitnehmers nicht in der schnelleren Durchführung einzelner Arbeitselemente, sondern in unterschiedlichen Arbeitsaufgaben mit nicht festgestellten Arbeitsunterbrechungen begründet ist.

\subsection{Arbeitszeitbedarf von Klein- und Großgruppe im Wartebereich}

Der Arbeitszeitbedarf ist in den nachfolgenden Teilabschnitten nach der Routine- und Sonderarbeit und dem Gesamtarbeitszeitbedarf der modellierten Modellbetriebe nach den beiden verschiedenen Haltungssystemen dargestellt und diskutiert.

\subsubsection{Routinearbeiten}

$\mathrm{Zu}$ den Routinearbeiten der Gesamtarbeit zählten alle Arbeitsvorgänge, welche täglich erledigt wurden. Diese beliefen sich auf 70,9 \% des Gesamtarbeitszeitbedarfs bei der Kleingruppe und auf 67,9\% des Gesamtarbeitszeitbedarfs bei der Großgruppe für den Bestand von 140 Zuchtsauen im Wartebereich, basierend auf den mittleren Leistungsdaten der Untersuchungsbetriebe. Diese setzten sich bei der Klein- und Großgruppe aus den verschiedenen Arbeitsvorgängen Füttern, Tierkontrolle, Misten und Dokumentation, die in Tabelle 2 im Arbeitszeitbedarf vergleichend dargestellt sind, zusammen.

Das Misten verursachte mit 55,7 \% bei der Kleingruppe als auch mit 66,8 \% bei der Großgruppe den höchsten
Arbeitszeitbedarf je Sau und Jahr, gefolgt vom Füttern, innerhalb der Routinearbeit. Die größten, teils signifikanten, haltungssystembedingten Unterschiede im Arbeitszeitbedarf innerhalb der Routinearbeit im Wartebereich wurden bei den Arbeitsvorgängen Füttern mit einem höheren Arbeitszeitbedarf von $135 \%$ (in AKmin/Sau/Jahr), der Tierkontrolle von 34,0 \% und dem Misten von 16,8 \% bei der Kleingruppe gegenüber der Großgruppe festgestellt (Tabelle 2).

Dieser kam aufgrund verschiedener Teilarbeitsvorgänge sowie -elemente in der Häufigkeit zustande. Bei den Wegstrecken der beiden Haltungssysteme wurden durch die Abrufstation in der Großgruppe bei jedem Fütterungsvorgang 90,1 m weniger als bei der Kleingruppe (152 m) zurückgelegt. Bei der Großgruppe wurden nur 2 statt 4 Türen benutzt und 4 statt 12 Sauentränken bedient. Durch die Abrufstation wurden auch 4 Hebel für das Auslösen der Futterdosierer erspart. Der kurze Kontrollblick am PC von täglich 5 APs bei der Großgruppe erhöhte den Arbeitszeitbedarf unwesentlich, um durchschnittlich 0,10 AKmin/Sau/Jahr. Blumauer (2004) ermittelte, dass Betriebe mit 80 Zuchtsauen und automatischer Fütterung durchschnittlich einen Arbeitszeitaufwand von 2,70 APh/Sau/Jahr für die Fütterung im Deck- und Wartebereich benötigen. Riegel und Schick (2006) gaben hingegen in ihrer Arbeit bei einem konventionellen Betrieb mit Abruffütterung einen Arbeitszeitbedarf von etwa $4 \mathrm{AKh} / \mathrm{Sau} / \mathrm{Jahr}$ an. Trotz des angegebenen hohen Automatisierungsgrades der Betriebe der Vergleichsstudien lag der Arbeitszeitbedarf der beiden Modellbetriebe unter den Literaturangaben. Als Verbesserungsvorschlag zur Senkung des Arbeitszeitbedarfs für das Füttern sind im Deckzentrum bei beiden Haltungssystemen und im Wartebereich der Kleingruppe ein automatisches Befüllen und Auslösen der Futterdosierer mit Trockenfutter, durch eine

Tabelle 2. Arbeitszeitbedarf (AKmin/Sau/Jahr) für die Routinearbeit im Wartebereich der Modellbetriebe mit 140 Zuchtsauen nach der Gruppengröße (Klein- oder Großgruppehaltung), mittleren Leistungsdaten der Untersuchungsbetriebe und Signifikanzniveaus

Table 2. Working time requirement (MPmin/sow/year) for routine work in the waiting area of the model farms with 140 sows depending on group size (small or large group), medium performance date of trial farms and significant levels

\begin{tabular}{|c|c|c|c|}
\hline \multirow[b]{2}{*}{ Arbeitsvorgänge } & \multicolumn{2}{|c|}{ Arbeitszeitbedarf (AKmin/Sau/Jahr) } & \multirow{2}{*}{$\begin{array}{c}\begin{array}{c}\text { Signifikanzniveau } \\
\text { (t-test) }\end{array} \\
\text { p-Wert }\end{array}$} \\
\hline & Kleingruppenhaltung & Großgruppenhaltung & \\
\hline Füttern & 48,7 & 20,7 & 0,261 \\
\hline Tierkontrolle & 13,9 & 10,4 & $<0,001$ \\
\hline Misten & 85,0 & 72,8 & $<0,001$ \\
\hline Dokumentation & 5,09 & 5,09 & \\
\hline Summe (Routinearbeit) & 153 & 109 & 0,025 \\
\hline
\end{tabular}


elektronische Steuerung, vorzusehen. Diese beschränkt die Tätigkeit Füttern, bei eingeschalteten Sauentränken während des Fütterungsvorgangs, auf die technische Überwachung der Fütterungsanlage. Für die Tierkontrolle, die 9,10 \% bei der Kleingruppe und 9,54 \% bei der Großgruppe der Routinearbeit je Sau und Jahr ausmachte, ergab sich zwischen den Gruppengrößen ein hoch signifikanter Unterschied. Tamtögl (2010) ermittelte einen Arbeitszeitbedarf für die Tierkontrolle von 0,22 AKh/Sau/ Jahr. Unter Berücksichtigung, dass in den Modellen nur der Deck- und Wartebereich berechnet wurden, deckt sich diese Angabe mit den Ergebnissen der Modelle.

\subsubsection{Sonderarbeiten}

$\mathrm{Zu}$ den Sonderarbeiten zählten alle Arbeitsvorgänge und deren -elemente, welche während eines einzigen Produktionszyklus nur einmal oder wenige Male zu erledigen waren. Dazu zählten die künstliche Besamung, das Umstallen, die Trächtigkeitskontrolle, Futterbereitung, das Problemsauen separieren, die Sauenbehandlung, Stallreinigung, Wartung, Reparatur und Vermarktung. Deren ermittelter Arbeitszeitbedarf (APmin je Sau und Jahr) gemäß mittleren Leistungsdaten der Untersuchungsbetriebe ist in Tabelle 3 nach den Haltungsverfahren und Signifikanzen aufgezeigt. Sie beliefen sich auf 29,7 \% für die Kleingruppe und 32,1 \% für die Großgruppe des Gesamtarbeitszeitbedarfs im Wartebereich. Martetschläger (2007) ermittelte für einen Bestand von 600 produktiven Sauen einen Arbeitszeitbedarf für die Sonderarbeiten von $0,30 \mathrm{AKh} / \mathrm{Sau} / \mathrm{Jahr}$, die $15 \%$ des Gesamtarbeitszeitbedarfs ausmachten. Dieser Arbeitszeitbedarf entsprach weniger als die Hälfte bis ein Drittel beider Modellbetriebe der untersuchten Haltungssysteme und belief sich nur auf die Hälfte des modellierten Arbeitszeitbedarfs des modellierten Untersuchungsbetriebes mit einem Bestand mit 630 Zuchtsauen und Großgruppe.

Die künstliche Besamung und die Futterbereitung veranschlagten zusammen etwa die Hälfte des Arbeitszeitaufwandes der gesamten Sonderarbeiten und unterschieden sich nicht nach den Haltungssystemen. Unterschiede im Arbeitszeitbedarf von Sonderarbeiten nach den untersuchten Haltungssystemen lagen für die Arbeitsvorgänge Umstallen, Problemsauen separieren, Stallreinigung, Wartung, Reparatur und Vermarktung (Altsau) vor. Nur die Arbeitsvorgänge Umstallen der Sauen vom Deckzentrum in den Wartestall sowie in den Abferkelstall, die sich aus Elementen für Gehen, Türen öffnen und schließen sowie Futterdosierer einstellen oder Abrufstation programmieren zusammensetzten, unterschieden sich bei den untersuchten Haltungssystemen im Arbeitszeitbedarf hoch signifikant voneinander ( $\mathrm{t}$-Test, $\mathrm{p}<0,0001)$.

Die erstellten Regressionsmodelle für unterschiedliche Bestandsgrößen des Umstallens erwiesen sich aufgrund der hohen Bestimmtheitsmaße (Kleingruppe: 0,85; Großgruppe: 0,87 ) als geeignet. Auch auf Basis dieser lagen für die untersuchten Haltungssysteme hoch signifikante Arbeitszeitbedarfsunterschiede für das Umstallen im Wartebereich über die verschiedenen Bestandsgrößen vor (70 bis 1050 Sauen) (GLM, $\mathrm{p}<0,0001)$.

Tabelle 3. Arbeitszeitbedarf (AKmin/Sau/Jahr) für die Sonderarbeiten im Wartebereich der Modellbetriebe mit 140 Zuchtsauen nach Klein- oder Großgruppenhaltung, mittlere Leistungsdaten der Untersuchungsbetriebe und Signifikanzniveaus (t-Test)

Table 3. Working time requirement (MP/sow/year) for special work in the waiting area of the model farm with 140 sows by keeping in small or large group, medium performance data of trial farms and significant level ( $\mathrm{t}$-test)

\begin{tabular}{|c|c|c|c|}
\hline \multirow[b]{2}{*}{ Arbeitsvorgänge } & \multicolumn{2}{|c|}{ Arbeitszeitbedarf (AKmin bzw. APmin/Sau/Jahr) } & \multirow{2}{*}{$\begin{array}{c}\text { Signifikanzniveau (t-test) } \\
\text { p-Wert }\end{array}$} \\
\hline & Kleingruppenhaltung & Großgruppenhaltung & \\
\hline Künstliche Besamung & 15,30 & 15,30 & \\
\hline Umstallen & 3,50 & 2,23 & $<0,0001$ \\
\hline Trächtigkeitskontrolle & 1,03 & 1,03 & \\
\hline Futterbereitung & 14,7 & 14,7 & \\
\hline Problemsauen separieren & 1,98 & 3,27 & \\
\hline Sauenbehandlung & 5,00 & 5,40 & \\
\hline Stallreinigung & 8,57 & 5,02 & \\
\hline Wartung & 3,50 & 0,24 & \\
\hline Reparatur & 2,55 & 0,71 & \\
\hline Vermarktung (Altsau) & 6,12 & 3,63 & \\
\hline Summe (Sonderarbeit) & 62,4 & 51,6 & 0,033 \\
\hline
\end{tabular}


Beim Umstallen der Sauen vom Wartestall in den Abferkelstall bestanden ebenso zwischen den beiden Haltungssystemen signifikante Unterschiede im Arbeitszeitbedarf vor ( $t-$ Test, $p=<0,0001$, h. s.). In Tabelle 4 ist beispielhaft der Arbeitsvorgang Umstallen der Zuchtsauen vom Deckzentrum in den Wartestall mit den jeweils dazugehöri-gen Arbeitselementen detailliert dargestellt. Der Arbeits-zeitbedarf für das Umstallen je Sau und Jahr ergibt sich durch Summation über alle Arbeitselemente und deren Häufigkeiten (je durchgeführten Arbeitsprozess) sowie Einflussgrößen (z. B. Sauenbestand, Sauengruppen, jähr-liche Wurfanzahl) je Produktionsjahr. In den Modellbe-trieben mit der Bestandsgröße von 140 Sauen belief sich dieser für die Kleingruppe auf 3,50 AKmin/Sau/Jahr und für die Großgruppe auf 2,23 AKmin/Sau/Jahr. Bei die-sen Arbeitszeitbedarfswerten ist zu berücksichtigen, dass Arbeiten im Abferkelstall, wie zum Beispiel das Einstallen der
Sau in die Abferkelbucht, nicht berücksichtigt wurden. Die festgelegte Grenze war jeweils beim Ein- und Ausstallen das Überschreiten der Rüsselscheibe der Sau von der Tür der Abferkelbucht. Im Deckzentrum und Wartestall wurden alle Arbeitselemente des Ein- und Ausstallens uneingeschränkt zum Arbeitszeitbedarf aufsummiert. Der etwas geringere Arbeitszeitbedarf beim Umstallen der Großgruppe war darin begründet, dass zur Arbeitserledigung weniger Arbeitselemente mit geringerer Dauer, teils bedingt durch die größere Anzahl der zu treibenden Sauen je Gruppe, nötig waren. Auch durch das Arbeitselement ,Abrufstation programmieren', welches nur bei der Großgruppenhaltung vorkam, ergab sich im Modell für das Umstallen der Sauen bei der Großgruppe ein um fast drei Arbeitskraftstunden geringerer Arbeitszeitbedarf pro Jahr als bei der Kleingruppe. Haidn (1992) gab im Mittel 4,50 APmin/Sau für das Umstallen der Sauen vom Abferkelstall

Tabelle 4. Arbeitselemente des Arbeitsvorgangs Umstallen vom Deckzentrum in den Wartestall mit deren Häufigkeit und Arbeitszeitbedarf (AKcmin/ Sau/Jahr) nach Haltungssystem

Table 4. Work elements of the work operation rehousing from the breeding center to the waiting area with frequency and working time requirement (MPcmin/sow/year) due to the keeping system

\begin{tabular}{|c|c|c|c|c|c|c|}
\hline \multirow{3}{*}{ Arbeitselement } & \multirow{3}{*}{$\begin{array}{c}\text { Bezugsmenge (BM) } \\
\text { (Einheit) }\end{array}$} & \multirow{3}{*}{$\begin{array}{c}t \text { (Zeit) } \\
\text { pro BM } \\
\text { (AKcmin) }\end{array}$} & \multicolumn{2}{|c|}{ Kleingruppenhaltung } & \multicolumn{2}{|c|}{ Großgruppenhaltung } \\
\hline & & & $\begin{array}{c}\text { BM je } \\
\text { Umtrieb }\end{array}$ & $\begin{array}{l}\text { t pro Sau } \\
\text { und Jahr }\end{array}$ & $\begin{array}{c}\text { BM je } \\
\text { Umtrieb }\end{array}$ & $\begin{array}{l}\text { t pro Sau } \\
\text { und Jahr }\end{array}$ \\
\hline & & & & (AKcmin) & & (AKcmin) \\
\hline Stalltür öffnen & Vorgang & 7,37 & 1 & 0,89 & 1 & 0,89 \\
\hline $\begin{array}{l}\text { Gehen ohne Last } \\
\text { Schick [2005]) }\end{array}$ & m (Meter) & 1,70 & 248 & 51,0 & 155 & 32,0 \\
\hline $\begin{array}{l}\text { Futterdosierer } \\
\text { schließen }\end{array}$ & Vorgang & 3,63 & 20 & 8,81 & 20 & 8,81 \\
\hline $\begin{array}{l}\text { Besamungskorb- } \\
\text { bucht öffnen }\end{array}$ & Vorgang & 4,16 & 20 & 10,1 & 20 & 10,1 \\
\hline Buchttür öffnen & Vorgang & 7,86 & 2 & 1,91 & 1 & 0,95 \\
\hline $\begin{array}{l}\text { Sauen treiben in } \\
\text { Gruppe } 2 \text { bis } 5 \\
\text { Sauen }\end{array}$ & M & 1,44 & 148 & 25,9 & 122 & 21,2 \\
\hline $\begin{array}{l}\text { Fress-Liegestand } \\
\text { schließen }\end{array}$ & Vorgang & 5,50 & 20 & 13,3 & & \\
\hline Buchttür schließen & Vorgang & 8,50 & 2 & 2,06 & 1 & 1,03 \\
\hline $\begin{array}{l}\text { Fress-Liegestand } \\
\text { öffnen }\end{array}$ & Vorgang & 4,88 & 20 & 11,8 & & \\
\hline $\begin{array}{l}\text { Futterdosierer } \\
\text { öffnen }\end{array}$ & Vorgang & 3,24 & 20 & 7,85 & & \\
\hline $\begin{array}{l}\text { Futterdosierer } \\
\text { einstellen }\end{array}$ & Vorgang & 4,76 & 40 & 23,1 & & \\
\hline Stalltür schließen & Vorgang & 8,25 & 1 & 1,00 & 1 & 1,00 \\
\hline $\begin{array}{l}\text { Abrufstation } \\
\text { programmieren }\end{array}$ & Vorgang & 3,30 & & & 20 & 8,00 \\
\hline
\end{tabular}


in das Deckzentrum, 2,10 APmin/Sau vom Deckzentrum in den Wartestall und 7,80 APmin/Sau vom Wartestall wieder in den Abferkelstall pro Durchgang an. Für ein Jahr und gemäß heutiger Leistungsdaten waren dies etwa 6,00 APmin/Sau/Jahr. Dieser Arbeitszeitbedarf entsprach etwa dem Doppelten jenem der modellierten Betriebe dieser Studie und wurde durch einzelne Stallbereiche in verschiedenen Gebäuden der Betriebe verursacht. Laut Riegel und Schick (2006) lag der Arbeitszeitbedarf für das Umstallen der Sauen aus dem Abferkelstall in den Deckbereich bei 0,30 AKmin/Sau/Durchgang und jener in die anderen Stallbereiche bei 1,00 AKmin/Sau/Durchgang. Bei 2,43 Würfen/Sau/Jahr verursachte dies einen Arbeitszeitaufwand von $0,73 \mathrm{AKmin} / \mathrm{Sau} / \mathrm{Jahr}$ und jener für das Umstallen in Deckzentrum und Wartestall sowie in den anderen Stallbereichen belief sich auf $1 \mathrm{AKmin} / \mathrm{Sau} / \mathrm{Durchgang}$. Dies entsprach einem Arbeitszeitbedarf von 2,43 AKmin/Sau/Jahr für das Umstallen, welcher zwischen dem Bedarf der Großgruppehaltung mit 1,34 AKmin/Sau/Jahr und der Kleingruppehaltung mit 2,61 AKmin/Sau/Jahr lag. Um arbeitszeitsparend zu arbeiten, sollte bei Um- sowie Neubau für das Umstallen auf möglichst kurze Treibwege geachtet werden. Türen in den Wänden zwi-schen zwei Stallbereichen, zum Beispiel Deckzentrum und Wartestall, wirken sich vorteilhaft zur Reduzierung der Treibganglänge aus, anstatt der Verwendung eines Zent-ralganges mit mehreren Stichwegen oder Stallbereiche in getrennten Gebäuden.

Die künstliche Besamung als auch die Trächtigkeitskontrolle wurden bei beiden Haltungssystemen ident ausgeführt. Diese setzte sich aus dem Schätzwert für Besamungsportionen generieren und den Arbeitselementen Gehen mit Last $10 \mathrm{~kg}$ oder Wagen $100 \mathrm{~kg}$, Duldungsreflex prüfen, Besamungsbügel anbringen, Künstliche Besamung durchführen, Besamungsportion nochmals anstecken, Besamungskatheter entfernen, Besamungsbügel abnehmen und Sauen markieren zusammen. Die eruierten Arbeitselemente für die künstliche Besamung wurden für alle angeführten Arbeitselemente in die Datenbank PROOF Schweine nach Schick (2005) integriert. Die vorhandenen Arbeitselemente wurden, aufgrund unterschiedlicher Planzeitwerte im Vergleich zu den neu erhobenen Arbeitselementen, ersetzt. Der Arbeitszeitbedarf für die Besamung belief sich 15,3 AKmin/Sau/Jahr. Ein Großteil davon entfiel auf das Generieren der Besamungsportionen. Pro Durchgang einer Sauengruppe wurden für die Bestellung und Abholung ein mittlerer Arbeitszeitaufwand von 90 APmin geschätzt. Ohne die Generierung der Besamungsportionen betrug die Besamungsarbeit im
Stall 4,40 AKmin/Sau/Jahr bei zweimaliger Besamung pro Durchgang. Haidn (1992) nannte für die Durchführung der künstlichen Besamung einen Arbeitszeitbedarf von 4,20 APmin je Besamung. Damit liegt dieser angegebene Wert um ein Vielfaches höher als jener dieser Studie. Bei den Modellbetrieben wurde auch die Gruppenabferkelung unterstellt, die auch die gleichzeitige Besamung der Sauen einer Gruppe voraussetzt und sehr wahrscheinlich auf den Untersuchungsbetrieben von Haidn (1992) nur teilweise gängige Praxis war. Durch diese Vorgehensweise kann die Besamung aller Sauen gleichzeitig erfolgen und sehr effizient durchgeführt werden. Durch ein Anordnen der Sauen nebeneinander im Deckzentrum können auch Arbeitswege auf ein Minimum beschränkt werden und durch die Einzelhaltung entfiel das Treiben und Fixieren. Bei der Trächtigkeitskontrolle mit Sicht betrug der Arbeitszeitbedarf 0,20 AKmin/Sau/Jahr. Bei der Trächtigkeitskontrolle mit dem Trächtigkeitsdetektor wurden 0,40 AKmin/Sau/Jahr aufgewendet und bei Verwendung eines Ultraschallgerätes rund 1,00 AKmin/Sau/Jahr. Haidn (1992) führte bei der Verwendung eines Ultraschallgerätes 1,30 APmin pro Vorgang an, der bei 2,43 Würfen pro Sau pro Jahr, wie im Modell angenommen, dem dreifachen Arbeitszeitbedarf der Trächtigkeitskontrolle mit Ultraschall entsprach. Die Verwendung eines Ultraschallgerätes bedingte den größten Arbeitszeitbedarf der drei Methoden, allerdings konnten umrauschende Sauen zuverlässig festgestellt werden. Mit großer Wahrscheinlichkeit ist diese Methode in Zukunft in der Ferkelproduktion bevorzugt zu wählen.

Der mittlere Arbeitsaufwand der Sonderarbeiten Futterbereitung, Separieren von Problemsauen, Sauenbehandlung, Stallreinigung, Wartung, Reparatur und Vermarktung wurden über die erfragten Schätzwerte ermittelt, da ein messtechnisches Erfassen in den Untersuchungsbetrieben nicht möglich war. Die Futterbereitung unterschied sich nicht zwischen der Klein- und Großgruppenhaltung und betrug im Mittel 18,6 APmin/t Futter oder 14,7 APmin/Sau/Jahr. Das Separieren von Problemsauen nahmen Betriebe mit Kleingruppenhaltung 1,50 Mal im Monat vor und wendeten hierfür 6,00 APmin/Sau auf. Betriebe mit Großgruppenhaltung führten es 1,17 Mal im Monat zu je 7,60 APmin/Sau durch. Der Arbeitszeitaufwand für das Separieren von Problemsauen bei der Kleingruppenhaltung betrug folglich 1,98 APmin/Sau/Jahr und bei jener der Großgruppe 3,27 APmin/Sau/Jahr. Für die Sauenbehandlung, welche die tierärztlichen Impfungen umfasste und zweimal im Jahr durchgeführt wurde, 
betrug der Arbeitszeitaufwand bei der Kleingruppe 5,00 und bei der Großgruppe 5,40 APmin/Sau/Jahr. Tamtögl (2010) erzielte für das Behandeln der Sauen mit 0,07 $\mathrm{APh} / \mathrm{Sau} / \mathrm{Jahr}$ ein ähnliches Ergebnis der Kleingruppe dieser Studie. Bei der Stallreinigung unterschieden sich die beiden Haltungssysteme mit unterschiedlichen Stalleinrichtungen und damit verbundener Reinigungsarbeit. Für die Kleingruppenhaltung wurde ein Arbeitszeitaufwand, bei einem zweieinhalbmaligen Waschen und anschließender Desinfektion der Stallbereiche, von 8,57 APmin/Sau/ Jahr und für die Großgruppenhaltung von 5,02 APmin/ Sau/Jahr ermittelt. Im Mittel dauerte das Waschen in der Kleingruppenhaltung etwa doppelt so lang wie in der Großgruppenhaltung, bedingt durch die Konstruktionsunterschiede. Die Erhebungsbetriebe der Kleingruppenhaltung führten siebenmal öfter eine technische Wartung durch, welche im Durchschnitt etwa zweimal so lange dauerte als bei jener der Großgruppe. Der Arbeitszeitaufwand für die Wartung betrug bei der Kleingruppehaltung 3,50 APmin/Sau/Jahr und 0,24 APmin/Sau/Jahr bei jener der Großgruppe. Die Betriebsleiter der Kleingruppenhaltung gaben eine vierfach höhere Anzahl an Reparaturen an, welche allerdings mit je einer Stunde etwa gleich lang dauerten als bei den Betrieben mit Großgruppenhaltung.
Der Arbeitszeitaufwand für Reparaturen verhielt sich folglich für die Kleingruppe erheblich höher. Die Vermarktung setzte sich aus dem Verkaufsgespräch, der Lieferung der Tiere und die Verrechnung zusammen und verursachte für die Kleingruppen einen beinahe doppelt so hohen Arbeitszeitaufwand pro Sau und Jahr. Die drei letztgenannten Arbeitsvorgänge der Sonderarbeiten wurden in bisherigen Studien nicht erhoben, weshalb eine Diskussion nicht möglich war.

\subsubsection{Gesamtarbeitszeitbedarf}

Der Gesamtarbeitsbedarf im Wartebereich, gemäß neuer Arbeitselemente und -vorgänge obiger Routine- und Sonderarbeiten sowie Leistungsdaten der Untersuchungsbetriebe, wurde modelliert in der Datenbank PROOF Schweine. Dieser belief sich für eine Bestandsgröße von 140 Sauen, die typisch für waschsende Betriebe in Österreich ist, für die Kleingruppe auf 3,58 AKh/Sau/Jahr und die Großgruppe auf 2,68 AKh/Sau/Jahr. Für die Kleingruppenhaltung verhielt sich dieser signifikant höher als in der Großgruppenhaltung ( $\mathrm{n}=16$; $\mathrm{t}$-Test; $\mathrm{p}=0,026$; Abbildung 1). Dieser nahm bei beiden, der Klein- und Großgruppenhaltung, mit zunehmender Bestandsgröße ab. Hohe Bestimmtheitsmaße für Klein- $\left(\mathrm{R}^{2}=0,90\right)$ und

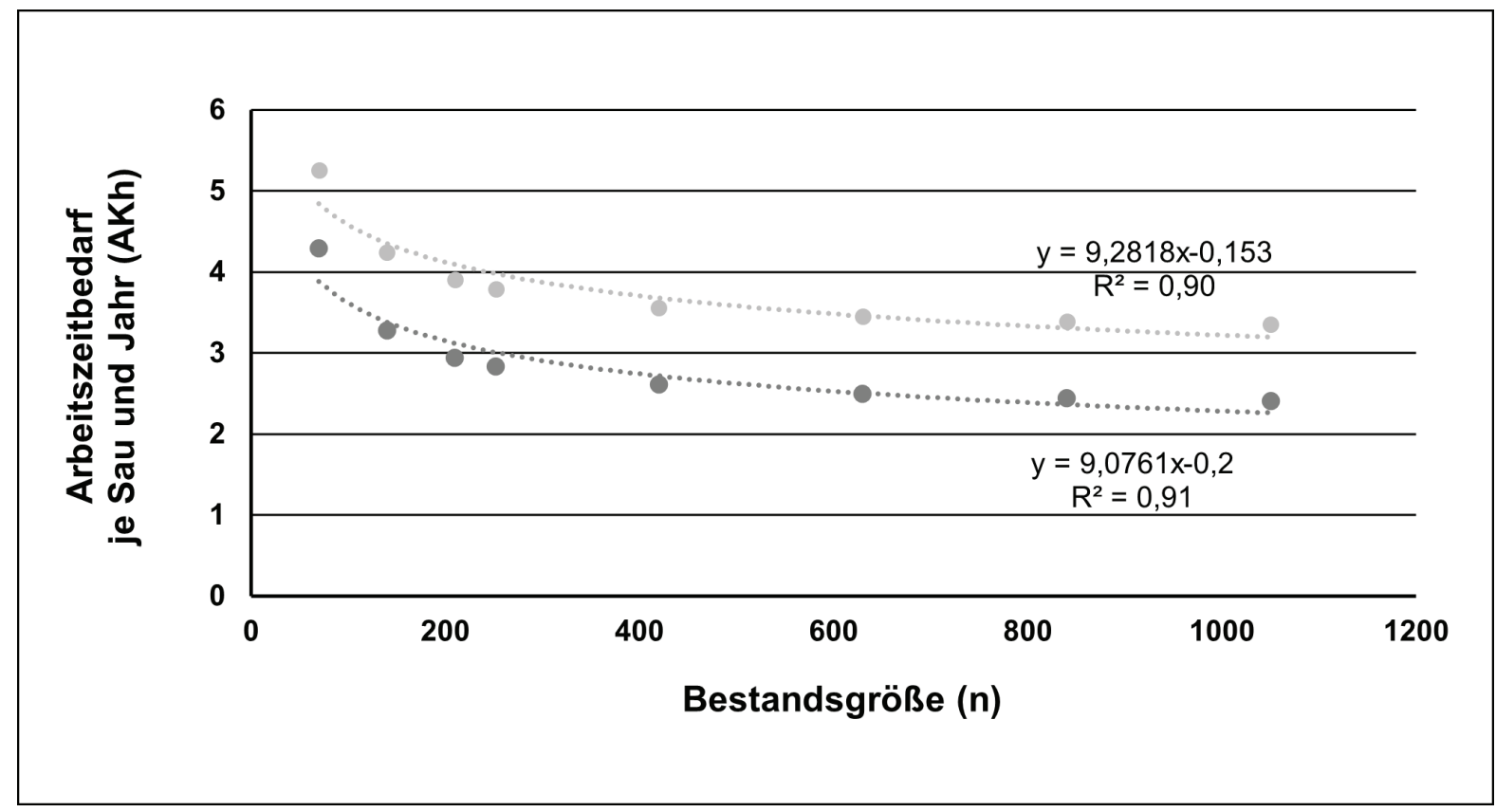

Abbildung 1. Gesamtarbeitszeitbedarf (AKh/Sau/Jahr) im Wartebereich nach Haltungssystemen, mittleren Leistungsdaten der Untersuchungsbetriebe und Bestandsgrößen von 70 bis 1050 Zuchtsauen

Figure 1. Total working time requirement ( $\mathrm{MPh} /$ sow/year) in the waiting area for housing systems, medium performance data of trial farms and stock sizes of 70 to 1050 breeding sows 
Großgruppe $\left(\mathrm{R}^{2}=0,91\right)$ zeigten eine gute Anpassung der Regressionsgleichungen an den vorliegenden Daten. Nach Haidn (1992), Blumauer (2004) und Handler et al. (2006) lag der durchschnittliche Arbeitszeitaufwand sowie -bedarf bei 24,1 APh/Sau/Jahr (80 Zuchtsauen), 18,1 $\mathrm{APh} / \mathrm{Sau} / \mathrm{Jahr}$ (129 Zuchtsauen) und 34,4 AKh/Sau und Jahr (durchschnittliche österreichische Standardarbeitszeitbedarf der Zuchtsauenhaltung). Bei Abzug der Arbeitsanteile für Abferkel- und Säugephase sowie Ferkelaufzucht im Ausmaß von etwa $60 \%$ beliefen sich diese auf 9,64 und 7,24 APh/Sau/Jahr sowie 12,9 AKh/Sau/Jahr. Folglich ist der Gesamtarbeitszeitbedarf des Wartebereichs der Modellbetriebe, die im Tierbestand den wachsenden österreichischen Zuchtsauenbetrieben entsprechen, bedingt durch Optimierungen der Haltungssysteme und Wachstum, um das Zwei- bis Dreifache niedriger als jener konventioneller Zuchtsauenbetriebe der 90er und 2010er Jahre und spiegelt den Trend einer Abnahme des Arbeitszeitbedarfs je Sau und Jahr durch fortschreitende technische Entwicklungen, insbesondere Zunahme der Automatisierung, und den fortschreitenden Strukturwandel in der Landwirtschaft wider.

\section{Schlussfolgerungen}

Gemäß obiger Ergebnisse ist zu folgern, dass mit dem technischen Fortschritt periodisch wiederkehrend es nötig ist, aktuelle Planungsdaten zu generieren. Durch ein Erstellen von Arbeitszeitmodellen können Aussagen über den Arbeitszeitbedarf im Wartebereich über verschiedene Bestandsgrößen getroffen werden, wie dies exemplarisch für den Gesamtarbeitszeitbedarf aufgezeigt wurde. Der Gesamtarbeitszeitbedarf im Wartebereich je Sau und Jahr variierte nicht nur mit der Bestandsgröße, sondern auch mit dem Haltungssystem Groß- und Kleingruppe wesentlich. Über ein Optimieren der Arbeitserledigung lässt sich systembezogen der Gesamtarbeitszeitbedarf im Wartebereich je Sau und Jahr stets senken. Dies kann durch die Herdengröße, das Management, einen höheren Automatisierungsgrad und routinierte Arbeitsweisen von Betriebsleitern erzielt werden.

\section{Danksagung}

Die Autoren danken dem KTBL (Kuratorium für Technik und Bauwesen in der Landwirtschaft e. V.) und den teilnehmenden Landwirten, welche diese Studie ermöglicht haben.

\section{Literatur}

Auernhammer, H. (1976): Eine integrierte Methode zur Arbeitszeitanalyse, Planzeiterstellung und Modellkalkulation landwirtschaftlicher Arbeiten, dargestellt an verschiedenen Arbeitsverfahren. Dissertation, Technische Universität München.

Blumauer, E. (2004): Arbeitswirtschaftliche Situation in der oberösterreichischen Ferkelproduktion. In: Schick, M. (Hrsg.): 14. Arbeitswissenschaftliches Seminar des VDI-MEG-Arbeitskreises Arbeitswissenschaften und Landbau. FAT-Schriftenreihe 62, FAT Tanikon, Ettenhausen, S. 65-74.

Fontana, M.-C., Fitzli, D., Koebel, K., Wegmann, B., Duffner, M. und M. Schaub (2016): Zusammenarbeit von Wissenschaft und Zivilgesellschaft - Bestandsaufnahme und Bedarfsanalyse. Schlussbericht, 23. März 2016, econcept AG, Zürich.

Fricke, W. (2004): Statistik in der Arbeitsorganisation, Hanser-Verlag, Darmstadt.

Haidn, B. (1992): Arbeitswirtschaftliche Untersuchungen und Modellkalkulati-onen in der Zuchtsauenhaltung. Dissertation, Technische Universität München.

Haidn, B., Schleicher, T. und J. Macuhová (2007): Bavarian animal welfare pilotfarms - Labor input by comparison. Agricultural Engineering Research 13, 151-158.

Handler, F., Stadler, M., Blumauer, E. (2006): Standardarbeitszeitbedarf in der österreichischen Landwirtschaft - Ergebnis der Berechnung der einzelbetrieblichen Standardarbeitszeiten. HBLA Francisco Josephinum, BLT Biomass - Logistics - Technology, Report Nr. 48, Wieselburg.

Hoy, S., Bauer, J. und B. Feller (2015): Gruppenbildung von Sauen, DLG-Merkblatt 408, 1. Auflage, DLG e. V., Frankfurt/Main, 4, 8, 12.

DMC (2019): https://www.dmc-group.com/dmcortim/produkte/zeitstudien/ortimzeit. Abgerufen am 22.06.2019.

Martetschläger, R. (2007): Arbeitszeitvergleich von Abferkelbuchttypen mit und ohne Fixierung der Sau. Diplomarbeit, Universität für Bodenkultur Wien.

Martel, G., Dourmad, J. Y. und B. Dedieu (2008): Do labour productivity and preferences about work load distribution affect reproduction management in pig farms, Livestock Science, 116 (1), 96-107. 
ÖKL-Arbeitskreis Landwirtschaftsbau (2016):Zuchtsauen Wartestall, Stallbereiche für Gruppieren, Decken und Trächtigkeit, ÖKL-Merkblatt Nr. 16, 3. Aufl., 24 S.

Pötz, P. (2012): Ermittlung des Arbeitszeitbedarfs der Welser Abferkelbucht. Masterarbeit, Universität für Bodenkultur Wien.

Quendler, E., Martetschläger, R.; Baumgartner, J., Koller, M., Schick, M. and J. Boxberger, Pötz, P., Hagmüller, W., Kogler, R., Boxberger, J. und I. Mösenbacher (2007): Logging of Time Elements with Digital Video Technology in Baby Piglet Production, CIGR Ejournal, Vol. IX, 1-8.

Quendler, E., Podiwinsky, C., Martetschläger, R., Helfendörfer, V., Baumgartner, J., Winckler, C. und J. Boxberger (2010): Arbeitswirtschaftliche und ökonomische Analyse verschiedener Abferkelsysteme. Die Bodenkultur 61, 29-37.

Quendler, E., Pötz, P., Hagmüller, W., Kogler, R. und J. Boxberger (2013): Determination of the working time requirement for suckling sows in the pen of Wels, Journal of Agricultural Engineering, Volume XLIV(S2): e 128, 641-644.

Riegel, M. und M. Schick (2006): Arbeitszeitbedarf und Arbeitsbelastung in der Schweinehaltung - Ein Vergleich praxisüblicher Systeme in Zucht und Mast. Agroscope, FAT Tänikon, FAT-Berichte Nr. 650.
Riegel, M., Schick, M., Klöble, U. und S. Fritzsche (2009): Arbeitszeitbedarf in der ökologischen Zuchtsauenhaltung. Landtechnik 64, 212-214.

Schulte-Sutrum, R. (2018): Fütterungsanlagen für Schweine, Mischen und Transportieren, DLG-Merkblatt 361, DLG e. V., Frankfurt/Main.

Schick, M. (2005): Methodenpapier Arbeitswirtschaft, Kapitel: Datenerfassung, -aufbereitung, Statistik. KTBLSchrift, Agroscope FAT Tänikon. Statistik Austria (2018): Viehbestand. http://www.statistik.at/web_de/statistiken/ wirtschaft/land_und_forstwirtschaft/viehbest and_tierische_erzeugung/viehbestand/index.html. Abgerufen am 08.01.2019.

Tamtögl, M. (2010): Ermittlung des Arbeitszeitbedarfs unter Berücksichtigung der Mensch-Tier-Beziehung in der biologischen Ferkelproduktion. Diplomarbeit, Universität für Bodenkultur Wien.

Weiß, J., Pabst W., Strack, K. und S. Granz (2005): Tierproduktion. 13. Aufl., Parey Verlag, Stuttgart.

Ziron, M. (2010): Fütterungstechnik in der Sauenhaltung, DLG-Merkblatt 359, DLG e. V., Frankfurt/Main.

Ziron, M. und T. Scholz (2018): Fütterungstechnik für tragende Sauen in der Gruppenhaltung, 1. Auflage, DLG e. V., Frankfurt/Main. 